\title{
Geometry effects in confined space
}

\author{
Wu-Sheng Dai * \\ Department of Physics, Tianjin University, Tianjin 300072, P. R. China \\ LuiHui Center for Applied Mathematics, Nankai University \& Tianjin University, Tianjin 300072, P. R. China \\ Mi Xie ${ }^{\dagger}$ \\ Department of Physics, Tianjin Normal University, Tianjin 3000\%4, P. R. China
}

\begin{abstract}
In this paper we calculate some exact solutions of the grand partition functions for quantum gases in confined space, such as ideal gases in two- and three-dimensional boxes, in tubes, in annular containers, on the lateral surface of cylinders, and photon gases in three-dimensional boxes. Based on these exact solutions, which, of course, contain the complete information about the system, we discuss the geometry effect which is neglected in the calculation with the thermodynamic limit $V \rightarrow \infty$, and analyze the validity of the quantum statistical method which can be used to calculate the geometry effect on ideal quantum gases confined in two-dimensional irregular containers. We also calculate the grand partition function for phonon gases in confined space. Finally, we discuss the geometry effects in realistic systems.
\end{abstract}

PACS numbers: 05.30.-d, 68.65.-k

\section{INTRODUCTION}

In statistical mechanics, for seeking the sum over all possible states, we always take the approximation that the volume of the system tends to infinity. In so doing we have lost all information about the geometry property of the system because in such an approximation the spectrum of single-particle states is continuous while the total number of states is independent of the shape of the boundary and simply proportional to the volume of the system. However, the properties of some systems found recently are shape dependent and sensitive to the topology [1-3]. In Ref. [4], we developed a method for calculating the effects of boundary and topology on ideal Bose and Fermi gases in confined space.

In the thermodynamic limit, one can replace the summation over states by an integral: $\sum_{s} \longrightarrow \int d \varepsilon \rho(\varepsilon)$, where

$$
\rho(\varepsilon)=V \frac{(2 \pi m)^{d / 2}}{h^{d} \Gamma(d / 2)} \varepsilon^{d / 2-1}
$$

is the density of states of a $d$-dimensional gas of dispersion $E=p^{2} /(2 m)$. However, if the system is enclosed in a finite volume, the structure of the phase space will be changed. In confined space, the spectrum of single-particle states will depend on the shape of the boundary. This is important if the thermal wavelength of particles is comparable to the size of the container, that is to say the replacement of the summation with the integral with the density of states Eq.(1) is a good approximation only when the volume is large enough so that the particle can not feel the boundary.

For taking into account geometry effects, in the preceding paper [4] we proposed that for the case of a twodimensional ideal quantum gas confined in an irregular container, the density of states, after neglecting the topological contribution, can be written as

$$
\rho(\varepsilon)=S \frac{2 \pi m}{h^{2}}-\frac{1}{4} L \frac{(2 m)^{1 / 2}}{h} \frac{1}{\varepsilon^{1 / 2}},
$$

where $S$ is the area and $L$ the perimeter of the container. Moreover, for the case of a three-dimensional ideal quantum gas in a long tube, of which all transverse cross-sections keep the same, the density of states is

$$
\rho(\varepsilon)=L_{z} S \frac{(2 \pi m)^{3 / 2}}{h^{3} \Gamma(3 / 2)} \varepsilon^{1 / 2}-\frac{1}{4} L_{z} L \frac{2 \pi m}{h^{2}} .
$$

\footnotetext{
*daiwusheng@tju.edu.cn

${ }^{\dagger}$ xiemi@mail.tjnu.edu.cn
} 
$S$ and $L$ here denote the area and perimeter of the transverse cross-section of the tube, respectively, and the length of the tube $L_{z}$ is made sufficiently large so that the $z$-component of the momentum $p_{z}$ can be considered to be continuous. The second terms of Eqs. (2) and (3) describe the contributions from the boundary.

In the present paper, for analyzing geometry effects, we will calculate some exact solutions for ideal gases and photon gases in various kinds of confined space, and provide an approximate result for phonon gases in confined space. By comparing these exact and approximate solutions with the result obtained in the thermodynamic limit $V \rightarrow \infty$, we can extract the information about boundary shape and topology. Furthermore, the validity of the method presented in Ref. [4] can be further justified by comparing it with these exact solutions.

It can be found that the corrections to the standard results which are on the order $\lambda / L$ where $\lambda$ is the thermal wavelength and $L$ denotes the linear size of the system. At the end of this paper, we will discuss such geometry effects in realistic systems.

In Sec. II, we discuss the method which will be used in this paper for calculating geometry effects. In Sec. III, we calculate the exact solutions for ideal gases in two-dimensional boxes, three-dimensional tubes and boxes. By comparing Eqs. (2) and (3) with these exact solutions, we can see that the densities of states given by Eqs. (2) and (3) are very good approximations for discussing ideal gases in confined space. Moreover, in this section, we also consider the geometry effect on the Fermi energy. In Sec. IV, we calculate the exact solutions for ideal gases in annular containers. The result shows no boundary effects in such systems even the scales of the systems are very small. In Sec. $\mathrm{V}$, we calculate the exact solutions for photon gases in confined space, and compare the thermodynamic quantities with the standard result of black-body radiation. In Sec. VI, we calculate an approximate result for phonon gases in confined space. Based on this result, we can analyze the boundary effect on the lattice specific heat. In Sec. VII, we analyze the geometry effects in realistic systems and compare the influence of geometry effects with the influence of fluctuations and interparticle interactions. The conclusions are summarized in Sec. VIII while some expressions of thermodynamic quantities are given in Appendix A.

\section{THE METHOD FOR CALCULATING GEOMETRY EFFECTS}

For an exact study of statistical mechanics in confined space, the geometry effect has to be reckoned in. To seek the exact solution of a grand potential, we need to find a method to perform the summation over all possible states because the energy spectrum is discrete in confined space. In the following we will solve the sum by using the Euler-MacLaurin formula [5]:

$$
\sum_{n=0}^{\infty} F(n)=\int_{0}^{\infty} F(n) d n+\frac{1}{2} F(0)-\frac{1}{2 !} B_{2} F^{\prime}(0)-\frac{1}{4 !} B_{4} F^{\prime \prime \prime}(0)+\cdots,
$$

where $B_{\nu}$ are Bernoulli Numbers: $B_{2}=1 / 6, B_{4}=-1 / 30, \cdots$.

The Euler-MacLaurin formula converts a sum to a series. Generally speaking, the Euler-MacLaurin formula can be used as an approximate method to solve sums approximately. However, in some special cases, this series has only a finite number of non-zero terms, i.e., $F^{(2 \nu+1)}(0)=0$ when $\nu$ is greater than a certain value (note that $B_{2 \nu+1}=0$ when $\nu>0$ ), and one can obtain the exact solution of the sum.

Moreover, a more general expression for the Euler-MacLaurin formula is

$$
\sum_{n=k}^{m-1} F(n)=\int_{k}^{m} F(n) d n-\frac{1}{2}[F(m)-F(k)]+\frac{B_{2}}{2 !}\left[F^{\prime}(m)-F^{\prime}(k)\right]+\frac{B_{4}}{4 !}\left[F^{\prime \prime \prime}(m)-F^{\prime \prime \prime}(k)\right]+\cdots,
$$

which will be used to discuss the phonon gas in confined space.

In statistical mechanics, one always replaces the summation over states by an integral in the thermodynamic limit $V \rightarrow \infty$. This treatment is equivalent to replacing the summation only by the integral on the right-hand side of Eq. (4) but neglecting the contribution from the rest terms. The information about the geometry of the system is contained in the summation over states since the structure of the spectrum of noninteracting particles is determined by the system geometry. The geometry information, however, will be lost after the replacement of the summation with the integral; in other words, the integral in Eq. (4) does not contain geometry information, that is to say, the rest terms in Eq. (4) correspond to geometry effects.

It is natural to expect that if there is no boundary, there is no boundary effect at all. No boundary implies periodic boundary conditions; in other words, there will be no boundary effect if we apply periodic boundary conditions. For example, we will show that there is no boundary effect in the system of an ideal gas in an annular container.

The prerequisite for applying the Euler-MacLaurin formula to solving the sum over states is that the energy spectrum of the system must be known. Whether one can obtain an exact solution or not depends on whether the series has a 
finite number of non-zero terms. If there are infinite non-zero $F^{(2 \nu+1)}(k)$, one obtains only an approximate solution. In the following we will calculate some exact and approximate solutions for ideal gases, photon gases and phonon gases in confined space.

\section{EXACT SOLUTIONS FOR IDEAL GASES IN CONFINED SPACE}

In this section, we first calculate some exact solutions for ideal gases in two- and three-dimensional confined space. Based on these exact solutions we then discuss the validity of the approximate method provided in Ref. [4], which can be used to calculate the geometry effect in the system with an irregular boundary. Moreover, as an application, we discuss the boundary effect on the Fermi energy.

\section{Exact solutions for ideal gases in two-dimensional cases}

In this part, by using the Euler-MacLaurin formula, we calculate the boundary effects on ideal gases in two twodimensional cases exactly: a) ideal gases in two-dimensional boxes, and b) ideal gases on the lateral surface of cylinders.

\section{a) Exact solutions for ideal gases in two-dimensional boxes}

Next we discuss the exact solution for ideal gases in two-dimensional boxes by using the Euler-MacLaurin formula.

The energy spectrum of a particle in a two-dimensional rectangular box of sides $L_{x}$ and $L_{y}$ is

$$
E\left(n_{x}, n_{y}\right)=\frac{\pi^{2} \hbar^{2}}{2 m}\left(\frac{n_{x}^{2}}{L_{x}^{2}}+\frac{n_{y}^{2}}{L_{y}^{2}}\right), \quad n_{x}, n_{y}=1,2 \cdots
$$

Then the grand potential of the system can be expressed as

$$
\ln \Xi=\mp \sum_{n_{x}=1}^{\infty} \sum_{n_{y}=1}^{\infty} \ln \left[1 \mp z e^{-\beta E\left(n_{x}, n_{y}\right)}\right] .
$$

In this equation and following, the upper sign stands for bosons and the lower sign for fermions. By use of the Euler-MacLaurin formula, we can perform the summation exactly:

$$
\begin{aligned}
\ln \Xi= & \mp \int_{0}^{\infty} \int_{0}^{\infty} d n_{x} d n_{y} \ln \left[1 \mp z e^{-\beta E\left(n_{x}, n_{y}\right)}\right] \pm \frac{1}{2} \int_{0}^{\infty} d n_{x} \ln \left[1 \mp z e^{-\beta E\left(n_{x}, 0\right)}\right] \\
& \pm \frac{1}{2} \int_{0}^{\infty} d n_{y} \ln \left[1 \mp z e^{-\beta E\left(0, n_{y}\right)}\right] \mp \frac{1}{4} \ln (1 \mp z) .
\end{aligned}
$$

We have

$$
\ln \Xi=\frac{S}{\lambda^{2}} h_{2}(z)-\frac{1}{4} \frac{L}{\lambda} h_{3 / 2}(z)+\frac{1}{4} h_{1}(z),
$$

where $S=L_{x} L_{y}$ is the area and $L=2\left(L_{x}+L_{y}\right)$ the perimeter of the box, $\lambda=h / \sqrt{2 \pi m k T}$ is the thermal wavelength, and the function

$$
h_{\sigma}(z)=\frac{1}{\Gamma(\sigma)} \int_{0}^{\infty} \frac{x^{\sigma-1}}{z^{-1} e^{x} \mp 1} d x
$$

equals the Bose-Einstein integral $g_{\sigma}(z)$ or the Fermi-Dirac integral $f_{\sigma}(z)$ in boson or fermion case, respectively.

Note that Eq. (9) is the exact solution for the grand potential. The first term is just the result obtained in the thermodynamic limit $S \rightarrow \infty$; the boundary effect is described by the second term which is proportional to the perimeter L. Comparing Eq. (9) with the corresponding approximate result given in Ref. [4] 


$$
\ln \Xi=\frac{S}{\lambda^{2}} h_{2}(z)-\frac{1}{4} \frac{L}{\lambda} h_{3 / 2}(z)+\frac{\chi}{6} h_{1}(z),
$$

we can see that the first two terms are completely equal to each other and the error introduced by the approximation appears only in the third term which describes the topology effect. The contribution from the topology effect is proportional to the Euler-Poincaré characteristic number $\chi$, in this case $\chi=1-r=1$ since $r$, the number of holes in this two-dimensional box, equals zero. From this, we can see the close analogy between the two results expressed in Eqs. (9) and (11). This close correspondence, notice that Eq. (9) is an exact solution, can be regarded as an evidence of the validity of the method given in Ref. [4].

\section{b) Exact solutions for ideal gases on the lateral surface of cylinders}

Consider an ideal gas confined on the lateral surface of a cylinder with radius $R$ and length $L$. Such a case is just the same as the ideal gas confined in a two-dimensional box of sides $L_{x}=2 \pi R$ and $L_{y}=L$, but the wavevector along $x$ is determined by periodic boundary conditions:

$$
k_{x}=\frac{n_{x}}{R}, \quad n_{x}=0, \pm 1, \pm 2, \cdots,
$$

while the wavevector along $y$ is determined by fixed-end boundary conditions:

$$
k_{y}=\frac{n_{y} \pi}{L}, \quad n_{y}=1,2, \cdots .
$$

The energy spectrum of a particle is

$$
E\left(n_{x}, n_{y}\right)=\frac{\hbar^{2}}{2 m}\left(k_{x}^{2}+k_{y}^{2}\right) .
$$

Then the grand potential can be calculated exactly by use of the Euler-MacLaurin formula:

$$
\begin{aligned}
\ln \Xi & =\mp \sum_{n_{x}} \sum_{n_{y}} \ln \left[1 \mp z e^{-\beta E\left(n_{x}, n_{y}\right)}\right] \\
& =\frac{S}{\lambda^{2}} h_{2}(z)-\frac{1}{4} \frac{L}{\lambda} h_{3 / 2}(z),
\end{aligned}
$$

where $S=2 \pi R L$ is the area of the surface, and $L=2 \cdot 2 \pi R=4 \pi R$ is the total length of the sides.

Furthermore, we can calculate the corresponding thermodynamic quantities directly, e.g., the specific heat

$$
\frac{C_{V}}{N k}=\sigma\left[2 \frac{h_{2}(z)}{h_{1}(z)}-\gamma \frac{h_{1}(z)}{h_{0}(z)}\right]-\frac{1}{\sqrt{N}} \frac{L}{\sqrt{S}} \sqrt{\sigma}\left[\frac{3}{16} \frac{h_{3 / 2}(z)}{h_{1}^{1 / 2}(z)}-\frac{1}{8} \gamma \frac{h_{1}^{1 / 2}(z) h_{1 / 2}(z)}{h_{0}(z)}\right],
$$

where

$$
\begin{aligned}
& \sigma=\left[\sqrt{1+\frac{1}{64 N} \frac{L^{2}}{S} \frac{h_{1 / 2}^{2}(z)}{h_{1}(z)}}-\frac{1}{8 \sqrt{N}} \frac{L}{\sqrt{S}} \frac{h_{1 / 2}(z)}{h_{1}^{1 / 2}(z)}\right]^{-2}, \\
& \gamma=\frac{1-\frac{1}{8 \sqrt{N}} \frac{L}{\sqrt{S}} \frac{h_{1 / 2}(z)}{h_{1}^{1 / 2}(z)} \frac{1}{\sqrt{\sigma}}}{1-\frac{1}{4 \sqrt{N}} \frac{L}{\sqrt{S}} \frac{h_{1}^{1 / 2}(z) h_{-1 / 2}(z)}{h_{0}(z)} \frac{1}{\sqrt{\sigma}}} .
\end{aligned}
$$

The following relation is used in the calculation:

$$
\frac{\partial z}{\partial T}=-\frac{z}{T} \frac{h_{1}(z)}{h_{0}(z)} \gamma
$$




\section{Exact solutions for ideal gases in three-dimensional cases}

We next calculate the boundary effects on ideal gases in two three-dimensional cases exactly: a) ideal gases in three-dimensional tubes with rectangular transverse cross-sections, and b) ideal gases in three-dimensional boxes.

\section{a) Ideal gases in three-dimensional tubes}

Consider an ideal gas enclosed in a three-dimensional tube with a rectangular transverse cross-section of sides $L_{x}$ and $L_{y}$. The $z$-component of the momentum $p_{z}$ is continuous since the length of the tube $L_{z}$ is made sufficiently large. In this case, the energy spectrum of a particle in the tube can be written as

$$
E\left(n_{x}, n_{y}, p_{z}\right)=\frac{\pi^{2} \hbar^{2}}{2 m}\left(\frac{n_{x}^{2}}{L_{x}^{2}}+\frac{n_{y}^{2}}{L_{y}^{2}}\right)+\frac{p_{z}^{2}}{2 m}, \quad n_{x}, n_{y}=1,2 \cdots
$$

The grand potential is

$$
\ln \Xi=\mp \int_{-\infty}^{\infty} \frac{L_{z} d p_{z}}{h} \sum_{n_{x}=1}^{\infty} \sum_{n_{y}=1}^{\infty} \ln \left[1 \mp z e^{-\beta E\left(n_{x}, n_{y}, p_{z}\right)}\right] .
$$

Here, we have converted the summation over $p_{z}$ into an integral since $p_{z}$ is continuous. Using the Euler-MacLaurin formula, we can perform the summations over $n_{x}$ and $n_{y}$ exactly:

$$
\ln \Xi=\frac{V}{\lambda^{3}} h_{5 / 2}(z)-\frac{1}{4} \frac{S}{\lambda^{2}} h_{2}(z)+\frac{1}{4} \frac{L_{z}}{\lambda} h_{3 / 2}(z) .
$$

Here the volume $V=L_{x} L_{y} L_{z}$ and the area of the lateral surface of the tube $S=L_{z} L$ where $L=2\left(L_{x}+L_{y}\right)$ is the perimeter of the transverse cross-section.

The first two terms of the exact solution Eq. (20) are consistent with the approximate result given in Ref. [4]:

$$
\ln \Xi=\frac{V}{\lambda^{3}} h_{5 / 2}(z)-\frac{1}{4} \frac{S}{\lambda^{2}} h_{2}(z)+\frac{\chi}{6} \frac{L_{z}}{\lambda} h_{3 / 2}(z)
$$

where $\chi$ is the Euler-Poincaré characteristic number of the transverse cross-section of the tube and in this case $\chi=1$. Comparing Eq. (20) with Eq. (21) we can see that, just as the two-dimensional case, the error only appears in the term corresponding to the topology effect.

\section{b) Ideal gases in three-dimensional boxes}

The energy spectrum of a particle in a three-dimensional box of sides $L_{x}, L_{y}$ and $L_{z}$ is

$$
E\left(n_{x}, n_{y}, n_{z}\right)=\frac{\pi^{2} \hbar^{2}}{2 m}\left(\frac{n_{x}^{2}}{L_{x}^{2}}+\frac{n_{y}^{2}}{L_{y}^{2}}+\frac{n_{z}^{2}}{L_{z}^{2}}\right), \quad n_{x}, n_{y}, n_{z}=1,2 \cdots
$$

The grand potential of an ideal gas in this box is

$$
\ln \Xi=\mp \sum_{n_{x}=1}^{\infty} \sum_{n_{y}=1}^{\infty} \sum_{n_{z}=1}^{\infty} \ln \left[1 \mp z e^{-\beta E\left(n_{x}, n_{y}, n_{z}\right)}\right] .
$$

By use of the Euler-MacLaurin formula, we can calculate the sum exactly:

$$
\begin{aligned}
\ln \Xi= & \mp \int_{0}^{\infty} \int_{0}^{\infty} \int_{0}^{\infty} d n_{x} d n_{y} d n_{z} \ln \left[1 \mp z e^{-\beta E\left(n_{x}, n_{y}, n_{z}\right)}\right] \pm \frac{1}{2} \int_{0}^{\infty} \int_{0}^{\infty} d n_{y} d n_{z} \ln \left[1 \mp z e^{-\beta E\left(0, n_{y}, n_{z}\right)}\right] \\
& \pm \frac{1}{2} \int_{0}^{\infty} \int_{0}^{\infty} d n_{x} d n_{z} \ln \left[1 \mp z e^{-\beta E\left(n_{x}, 0, n_{z}\right)}\right] \pm \frac{1}{2} \int_{0}^{\infty} \int_{0}^{\infty} d n_{x} d n_{y} \ln \left[1 \mp z e^{-\beta E\left(n_{x}, n_{y}, 0\right)}\right]
\end{aligned}
$$




$$
\begin{aligned}
& \mp \frac{1}{4} \int_{0}^{\infty} d n_{x} \ln \left[1 \mp z e^{-\beta E\left(n_{x}, 0,0\right)}\right] \mp \frac{1}{4} \int_{0}^{\infty} d n_{y} \ln \left[1 \mp z e^{-\beta E\left(0, n_{y}, 0\right)}\right] \\
& \mp \frac{1}{4} \int_{0}^{\infty} d n_{z} \ln \left[1 \mp z e^{-\beta E\left(0,0, n_{z}\right)}\right] \pm \frac{1}{8} \ln (1 \mp z) .
\end{aligned}
$$

Then we have

$$
\ln \Xi=\frac{V}{\lambda^{3}} h_{5 / 2}(z)-\frac{1}{4} \frac{S}{\lambda^{2}} h_{2}(z)+\frac{1}{16} \frac{L}{\lambda} h_{3 / 2}(z)-\frac{1}{8} h_{1}(z),
$$

where $V=L_{x} L_{y} L_{z}$ is the volume, $S=2\left(L_{x} L_{y}+L_{y} L_{z}+L_{z} L_{x}\right)$ the area of the surface and $L=4\left(L_{x}+L_{y}+L_{z}\right)$ the total length of the sides of the box.

We know that a box will change to a tube if its one side, e.g., $L_{z}$, is made very long. Assuming $L_{z} \gg L_{x}, L_{y}$, we then can take the approximation $S \simeq 2\left(L_{y}+L_{x}\right) L_{z}$ and $L \simeq 4 L_{z}$. It is easy to see that in this case Eq. (25) is the same as Eq. (20) except the last term which is negligibly small. In above discussions, we only concentrate on the case that the temperature of the system is higher than the critical temperature.

\section{The validity of the approximate method for calculating geometry effects with irregular boundaries}

In Ref. [4], for calculating geometry effects, we first expand the grand potential $\ln \Xi=\mp \sum_{s} \ln \left(1 \mp z e^{-\beta \epsilon_{s}}\right)$ as a series of $z e^{-\beta \epsilon_{s}}$, and then sum over all terms of the expansion after calculating these terms by use of the result given by Kac [6]. Clearly, the validity of this treatment depends on the value of the expansion parameter $z e^{-\beta \epsilon_{s}}$. Strictly speaking, however, when the fugacity $z>1$, such as in Fermi-Dirac statistics, the grand potential ln $\Xi$ can not be expanded in such a way. In the preceding paper [4] we perform the summation of the expansion of the grand potential without proving the convergence of the series, but, instead, we analyze the validity of the result by comparing it with the grand potential of an ideal quantum gas in free space and show that the grand potential in free space, whose validity is fully accepted, is just the zeroth-order approximation of our result. With the help of the exact solutions obtained above, we can make further improvement on the analysis of the validity of this treatment.

Comparing the results obtained by the approximate method developed in Ref. [4] with the exact solutions calculated in this section, we can see that this approximate method is valid for calculating the geometry effects in two- and threedimensional ideal gas systems. So far we have applied two methods to performing the summation in the grand potential for ideal gases in confined space: One is an approximate method [4] based on a mathematical work given by Kac [6]. Another is an exact one based on the Euler-MacLaurin formula. The approximate method applies equally well to the exact method in calculating the boundary effect, and the error introduced by the approximation appears only in the topology terms and is indeed small.

\section{The boundary effect on the Fermi energy}

Using the result obtained above, we can consider the boundary effect on the Fermi energy. From Eq. (25), we can obtain

$$
N=g\left[\frac{V}{\lambda^{3}} f_{3 / 2}(z)-\frac{1}{4} \frac{S}{\lambda^{2}} f_{1}(z)+\frac{1}{16} \frac{L}{\lambda} f_{1 / 2}(z)-\frac{1}{8} \frac{z}{z+1}\right]
$$

where $g$ is a weight factor that arises from the internal structure of the particles (the number of internal degrees of freedom).

The Fermi energy $\mu_{0}$ is the energy of the topmost filled level in the ground state of the $N$ electron system, so

$$
N=g\left[V \frac{1}{6 \pi^{2}}\left(\frac{\hbar^{2}}{2 m}\right)^{-3 / 2} \mu_{0}^{3 / 2}-\frac{1}{4} S \frac{1}{4 \pi}\left(\frac{\hbar^{2}}{2 m}\right)^{-1} \mu_{0}+\frac{1}{16} L \frac{1}{\pi}\left(\frac{\hbar^{2}}{2 m}\right)^{-1 / 2} \mu_{0}^{1 / 2}-\frac{1}{8}\right]
$$

The Fermi energy can be exactly calculated from this equation; however, for clarity we only calculate the next-toleading-order correction: 


$$
\begin{aligned}
\mu_{0} & \simeq \frac{\hbar^{2}}{2 m}\left(\frac{2}{g} 3 \pi^{2} \frac{N}{V}\right)^{2 / 3}+\frac{\hbar^{2}}{2 m} \frac{1}{2} \frac{S}{V}\left(\frac{3 \pi^{5}}{4 g} \frac{N}{V}\right)^{1 / 3} \\
& =\mu_{0}^{\text {free }}+\mu_{0}^{\text {boundary }} .
\end{aligned}
$$

Note that, for an electron gas the spin weight is $g=2$. Here $\mu_{0}^{\text {free }}$ is just the Fermi energy in free space, and $\mu_{0}^{\text {boundary }}$ reflects the influence of the boundary. It is easy to see that the existence of a boundary enhances the Fermi energy, and the Fermi energy increases with the decreasing size of the system. This result is not difficult to understand: In a finite size system, ground state energy and energy level spacing grow larger as the size of the system decreases.

\section{EXACT SOLUTIONS FOR IDEAL GASES IN ANNULAR CONTAINERS}

The exact solutions for ideal gases in annular containers can also be calculated with the help of the Euler-MacLaurin formula. In this section, we consider an ideal quantum gas confined in an one-dimensional ring of radius $R$. The energy of the particle in such an annular container is determined by periodic boundary conditions:

$$
\varepsilon_{n}=\frac{\hbar^{2}}{2 m R^{2}} n^{2}, \quad n=0, \pm 1, \pm 2, \cdots
$$

The grand potential of the ideal gas is

$$
\begin{aligned}
\ln \Xi & =\mp \sum_{n=-\infty}^{\infty} \ln \left(1 \mp z e^{-\beta \varepsilon_{n}}\right) \\
& =\mp \ln (1 \mp z) \mp 2 \sum_{n=1}^{\infty} \ln \left[1 \mp z e^{-\beta n^{2} \hbar^{2} /\left(2 m R^{2}\right)}\right] .
\end{aligned}
$$

The summation over the discrete parameter $n$ can be converted to an integral exactly, leading to

$$
\begin{aligned}
\ln \Xi & =\mp 2 \int_{0}^{\infty} d n \ln \left[1 \mp z e^{-\beta n^{2} \hbar^{2} /\left(2 m R^{2}\right)}\right] \\
& =\frac{L}{\lambda} h_{3 / 2}(z),
\end{aligned}
$$

where $L=2 \pi R$ is the perimeter of the container. There is no boundary effect because, for a ring, there is no boundary. This is an interest result. Generally speaking, for a finite perimeter $L$, the energy levels $\varepsilon_{n}$ are always discrete, so the

summation in Eq. (31) has to be performed approximately by replacing it by an integral: $\sum_{n=-\infty}^{\infty} \rightarrow \int_{-\infty}^{\infty} d n$. This replacement is based on the assumption that the momentum is continuous, which is valid only when $L \rightarrow \infty$. In many cases, such a treatment is an approximation that neglects the influence of the boundary; however, in an annular container this treatment gives an exact solution.

This result implies that, in a ring, e.g., an ideal electron gas in a conductor ring, even the perimeter is very small, then the energy level spacings are very large, we can replace the summation by an integral safely. The thermodynamic behavior of an ideal gas in a ring is the same as that in infinite free space. Generally, it is easy to prove that there is no boundary effect when one applies periodic boundary conditions, e.g., an ideal gas confined on a torus.

\section{EXACT SOLUTIONS FOR PHOTON GASES IN THREE-DIMENSIONAL CAVITIES}

In this section we calculate the exact solution for black-body radiation in confined space. From the electromagnetic field theory, it is found that the allowed values of $\mathbf{k}$ (the momentum $\mathbf{p}=\hbar \mathbf{k}$ ) in a rectangular cavity of sides $L_{x}, L_{y}$ and $L_{z}$ are

$$
k_{x}=\frac{\pi}{L_{x}} n_{x}, k_{y}=\frac{\pi}{L_{y}} n_{y}, k_{z}=\frac{\pi}{L_{z}} n_{z}, \quad n_{x}, n_{y}, n_{z}=0,1,2 \cdots,
$$

and the energy of a photon is 


$$
E\left(n_{x}, n_{y}, n_{z}\right)=c p=\pi \hbar c \sqrt{\frac{n_{x}^{2}}{L_{x}^{2}}+\frac{n_{y}^{2}}{L_{y}^{2}}+\frac{n_{z}^{2}}{L_{z}^{2}}}
$$

where $c$ is the velocity of light. Note that, $\left(n_{x}, n_{y}, n_{z}\right) \neq(1,0,0),(0,1,0),(0,0,1)$, or $(0,0,0)$, because if any two of the integers $n_{x}, n_{y}, n_{z}$ are zero, then all of the components of the electromagnetic field will be zero. Since the number of photons is indefinite, then the chemical potential of the system is identically zero, we have

$$
\ln \Xi=2\left[\sum_{n_{x}=1}^{\infty} \sum_{n_{y}=1}^{\infty} \sum_{n_{z}=1}^{\infty} A\left(n_{x}, n_{y}, n_{z}\right)+A(1,1,0)+A(1,0,1)+A(0,1,1)\right]
$$

where

$$
A\left(n_{x}, n_{y}, n_{z}\right)=-\ln \left\{1-\exp \left[-\beta E\left(n_{x}, n_{y}, n_{z}\right)\right]\right\}
$$

and the factor 2 comes from the two possible polarizations. The summation in Eq. (35) can be performed by using the Euler-MacLaurin formula:

$$
\begin{aligned}
\ln \Xi & =2\left[\frac{V}{\lambda^{3}} \frac{\Gamma(4)}{\Gamma(5 / 2)} \zeta(4)-\frac{1}{4} \frac{S}{\lambda^{2}} \frac{\Gamma(3)}{\Gamma(2)} \zeta(3)+\frac{1}{16} \frac{L}{\lambda} \frac{\Gamma(2)}{\Gamma(3 / 2)} \zeta(2)+A(1,1,0)+A(1,0,1)+A(0,1,1)\right] \\
& =V \frac{\pi^{2}}{45 \hbar^{3} c^{3}}(k T)^{3}-S \frac{1}{4 \pi \hbar^{2} c^{2}} \zeta(3)(k T)^{2}+L \frac{\pi}{48 \hbar c} k T+2[A(1,1,0)+A(1,0,1)+A(0,1,1)]
\end{aligned}
$$

where, as defined in Sec. III, $S$ is the area of the surface and $L$ the total length of the sides of the cavity, $\zeta(n)$ is the Riemann zeta function, $\lambda=2 \sqrt{\pi} \hbar c /(k T)$ is the mean wavelength of the photon.

For the total energy in the cavity, from Eq. (37) we obtain

$$
\begin{aligned}
U & =-\frac{\partial}{\partial \beta} \ln \Xi \\
& =V \frac{\pi^{2}}{15 \hbar^{3} c^{3}}(k T)^{4}-S \frac{\zeta(3)}{2 \pi \hbar^{2} c^{2}}(k T)^{3}+L \frac{\pi}{48 \hbar c}(k T)^{2}+2[B(1,1,0)+B(1,0,1)+B(0,1,1)]
\end{aligned}
$$

where

$$
B\left(n_{x}, n_{y}, n_{z}\right)=\frac{E\left(n_{x}, n_{y}, n_{z}\right)}{\exp \left[\beta E\left(n_{x}, n_{y}, n_{z}\right)\right]-1}
$$

It is easy to see that the first term of Eq. (38) corresponds to the Stefan law of black-body radiation: $U \propto T^{4}[7]$. It follows that the specific heat is

$$
C_{V}=k\left\{V \frac{4 \pi^{2}}{15 \hbar^{3} c^{3}}(k T)^{3}-S \frac{3 \zeta(3)}{2 \pi \hbar^{2} c^{2}}(k T)^{2}+L \frac{\pi}{24 \hbar c}(k T)+2[D(1,1,0)+D(1,0,1)+D(0,1,1)]\right\}
$$

where

$$
D\left(n_{x}, n_{y}, n_{z}\right)=\frac{\left[\beta E\left(n_{x}, n_{y}, n_{z}\right)\right]^{2} \exp \left[\beta E\left(n_{x}, n_{y}, n_{z}\right)\right]}{\left\{\exp \left[\beta E\left(n_{x}, n_{y}, n_{z}\right)\right]-1\right\}^{2}} .
$$

The contribution from the first term, which is proportional to the volume of the system, is just the result obtained under the hypothesis that $V \rightarrow \infty$ (the thermodynamic limit). The last three terms, which correspond to the contributions from certain states, are so small that can be neglected.

Furthermore, from the result obtained above we can learn that the density of states of a photon gas in confined space can be approximately written as

$$
\rho(\omega) d \omega=\left(V \frac{1}{\pi^{2} c^{3}} \omega^{2}-\frac{1}{4} S \frac{1}{\pi c^{2}} \omega\right) d \omega,
$$

where the second term describes the boundary effect. In other words, one can replace the summation over states $\sum_{s}$ by the integral $\int \rho(\omega) d \omega$. 


\section{PHONON GASES IN CONFINED SPACE}

In this section, we discuss the boundary effect on phonon gases. Solids are crystal lattices of atoms, each atom behaving as an coupled harmonic oscillator in the harmonic approximation. Phonons correspond to the normal modes of the system and behave as independent quantum oscillators. Assume that the system is a rectangular solid of dimensions $L_{x}, L_{y}, L_{z}$. The wavevector of the phonon $\mathbf{k}$ is restricted by fixed-end boundary conditions (not periodic boundary conditions because the system we considered is finite) to the values

$$
\left\{\begin{array}{l}
k_{x}=\frac{\pi}{L_{x}}, \frac{2 \pi}{L_{x}}, \cdots, \frac{N_{x} \pi}{L_{x}}, \\
k_{y}=\frac{\pi}{L_{y}}, \frac{2 \pi}{L_{y}}, \cdots, \frac{N_{y} \pi}{L_{y}}, \\
k_{z}=\frac{\pi}{L_{z}}, \frac{2 \pi}{L_{z}}, \cdots, \frac{N_{z} \pi}{L_{z}},
\end{array}\right.
$$

where $N_{i}, i=x, y, z$, is the number of atoms along $i$-axis, and the total number of atoms $N=N_{x} N_{y} N_{z}$. Strictly speaking, there are only $N_{i}-1$, not $N_{i}$, allowed values of $k_{i}$, because the solution for $N_{i} \pi / L_{i}$ permits no motion of any atom [8]; however, such an influence is so small that can be neglected safely.

The grand potential is

$$
\begin{aligned}
\ln \Xi & =-\beta\left(\phi_{0}+\sum_{i=1}^{3 N} \frac{1}{2} \hbar \omega_{i}\right)-\sum_{i=1}^{3 N} \ln \left(1-e^{-\beta \hbar \omega_{i}}\right) \\
& =-\beta\left[\phi_{0}+\sum_{i=1}^{N} \frac{1}{2} \hbar\left(c_{l}+2 c_{t}\right) k_{i}\right]-\sum_{i=1}^{N} \ln \left(1-e^{-\beta \hbar c_{l} k_{i}}\right)-2 \sum_{i=1}^{N} \ln \left(1-e^{-\beta \hbar c_{t} k_{i}}\right),
\end{aligned}
$$

where

$$
\omega_{l}=c_{l}|k|, \quad \omega_{t}=c_{t}|k|
$$

There are three polarizations for each value of $k$ : two of these are transverse and one longitudinal. $c_{t}$ is the velocity of transverse waves and $c_{l}$ the velocity of longitudinal waves. The summations in $\ln \Xi$ can be converted to a set of integrals by using the Euler-MacLaurin formula Eq. (5). Neglecting higher orders, we have

$$
\begin{aligned}
\ln \Xi= & -\frac{\phi_{0}}{k T}-N\left(\frac{\theta_{p}}{\theta_{0}}\right)^{3}\left[\frac{9}{8} \frac{\theta_{p}}{T}+3 \ln \left(1-e^{-\frac{\theta_{p}}{T}}\right)-D_{3}\left(\frac{\theta_{p}}{T}\right)\right] \\
& +\frac{S}{a^{2}} \frac{\pi}{16}\left[\frac{1}{3}\left(\theta_{l}+2 \theta_{t}\right) \frac{1}{T}+\ln \left(1-e^{-\frac{\theta_{l}}{T}}\right)-\frac{1}{2} D_{2}\left(\frac{\theta_{l}}{T}\right)+2 \ln \left(1-e^{-\frac{\theta_{t}}{T}}\right)-D_{2}\left(\frac{\theta_{t}}{T}\right)\right],
\end{aligned}
$$

where

$$
\theta_{p}=\frac{\hbar \omega_{p}}{k}, \theta_{l}=\frac{\pi \hbar c_{l}}{k a}, \theta_{t}=\frac{\pi \hbar c_{t}}{k a}
$$

and $a$ is the lattice constant. Like the Debye frequency in the Debye approximation we introduce the cutoff frequency $\omega_{p}$, which will return to the Debye frequency $\omega_{D}$ so that $\theta_{p}$ will return to the Debye temperature $\theta_{D}$ when one neglects the contribution from the boundary effect. As an approximation, we can take $\omega_{p} \approx \omega_{D}$. Here we define the function $D_{\sigma}(x)$ by

$$
D_{\nu}(x)=\frac{\nu}{x^{\nu}} \int_{0}^{x} d \xi \frac{\xi^{\nu}}{e^{\xi}-1}
$$

the case of $\nu=3$ gives the Debye function.

From Eq. (46), we can calculate the internal energy

$$
U=\phi_{0}+N k T\left(\frac{\theta_{p}}{\theta_{0}}\right)^{3}\left[\frac{9}{8} \frac{\theta_{p}}{T}+3 D_{3}\left(\frac{\theta_{p}}{T}\right)\right]-\frac{S}{a^{2}} k T \frac{\pi}{16}\left[\frac{1}{3}\left(\frac{\theta_{l}}{T}+2 \frac{\theta_{t}}{T}\right)+D_{2}\left(\frac{\theta_{l}}{T}\right)+2 D_{2}\left(\frac{\theta_{t}}{T}\right)\right],
$$

and the specific heat 


$$
C_{V}=3 N k\left(\frac{\theta_{p}}{\theta_{0}}\right)^{3}\left[4 D_{3}\left(\frac{\theta_{p}}{T}\right)-3 \frac{\theta_{p} / T}{e^{\theta_{p} / T}-1}\right]-\frac{S}{a^{2}} k \frac{\pi}{16}\left[3 D_{2}\left(\frac{\theta_{l}}{T}\right)-2 \frac{\theta_{l} / T}{e^{\theta_{l} / T}-1}+6 D_{2}\left(\frac{\theta_{t}}{T}\right)-4 \frac{\theta_{t} / T}{e^{\theta_{t} / T}-1}\right] .
$$

In the high temperature limit $\left(T \gg \theta_{p}, \theta_{l}, \theta_{t}\right.$ or $\left.x \ll 1\right)$, the internal energy and the specific heat are

$$
U=\phi_{0}+3 N k T\left(\frac{\theta_{p}}{\theta_{0}}\right)^{3}\left[1+\frac{1}{20}\left(\frac{\theta_{p}}{T}\right)^{2}\right]-\frac{S}{a^{2}} k T \frac{3 \pi}{16}\left[1+\frac{1}{72} \frac{\theta_{l}^{2}+2 \theta_{t}^{2}}{T^{2}}\right],
$$

and

$$
C_{V}=3 N k\left(\frac{\theta_{p}}{\theta_{0}}\right)^{3}\left[1-\frac{1}{20}\left(\frac{\theta_{p}}{T}\right)^{2}\right]-\frac{S}{a^{2}} k \frac{3 \pi}{16}\left[1-\frac{1}{72} \frac{\theta_{l}^{2}+2 \theta_{t}^{2}}{T^{2}}\right]
$$

where

$$
\theta_{0}^{3}=18 \pi^{2} \frac{N}{V}\left(\frac{\hbar}{k}\right)^{3}\left(\frac{1}{c_{l}^{3}}+\frac{2}{c_{t}^{3}}\right)^{-1}
$$

and $\theta_{0}$ equals the Debye temperature.

Correspondingly, in the low temperature region $\left(T \ll \theta_{p}, \theta_{l}, \theta_{t}\right.$ or $\left.x \gg 1\right)$ the internal energy and the specific heat become

$$
U=\phi_{0}+N k\left[\frac{9}{8}\left(\frac{\theta_{p}}{\theta_{0}}\right)^{3} \theta_{p}+\frac{3 \pi^{4}}{5} \frac{1}{\theta_{0}^{3}} T^{4}\right]-\frac{S}{a^{2}} k\left[\frac{\pi}{48}\left(\theta_{l}+2 \theta_{t}\right)+\frac{\pi}{4} \zeta(3)\left(\frac{1}{\theta_{l}^{2}}+\frac{2}{\theta_{t}^{2}}\right) T^{3}\right]
$$

and

$$
C_{V}=N k \frac{12 \pi^{4}}{5} \frac{T^{3}}{\theta_{0}^{3}}-\frac{S}{a^{2}} k \frac{3 \pi}{4} \zeta(3)\left(\frac{1}{\theta_{l}^{2}}+\frac{2}{\theta_{t}^{2}}\right) T^{2} .
$$

Each expression of the thermodynamic quantities has been divided into two parts: the first part, though it is also influenced by the boundary, is almost the result given by Debye; the second part, which is proportional to the area of the surface, describes the boundary effect.

Moreover, similar to the case of ideal gases in annular containers, we can easily prove that there is no contribution coming from the term which is proportional to the area of the surface of the system if we apply periodic boundary conditions.

\section{GEOMETRY EFFECTS IN REALISTIC SYSTEMS}

In this section, we shall briefly discuss the geometry effects in realistic systems. Such effects are usually negligible in the thermodynamic limit $(V \longrightarrow \infty)$. The subject which will be of most interest to us will be that of boundary effects on the thermodynamic property of the system in which the thermal wavelength of the particle is comparable to the system size, e.g., mesoscopic systems or optical microcavities.

1) Small size systems: In statistical physics, one often uses the thermodynamic limit $(V \longrightarrow \infty)$ as a convenient mathematical device in macroscopic limit. The system approaches the macroscopic limit once its size is much larger than the thermal wavelength. The size of a mesoscopic system is between microscopic and macroscopic scale. In mesoscopic systems, of course, many of rules in macroscopic physics, such as the thermodynamic limit (which neglects the boundary effect), may not hold. Many novel phenomena exist that are intrinsic to mesoscopic systems, e.g., size effects [9].

In realistic systems, the magnitude of boundary effects is determined by the ratio $\lambda / L$, where $\lambda$ is the thermal wavelength and $L$ denotes the linear size of the system. The thermal wavelength depends on two factors: the temperature of the system $T$ and the mass of the particle. For electrons, from room temperature to $10 K$ the thermal wavelength is in the range of about $4 \mathrm{~nm}$ to $24 \mathrm{~nm}$. This means that in a nanosystem the boundary effect becomes important and can not be neglected. Furthermore, in ultra-low-temperature physics, obviously, the boundary effect will become remarkable. Up to now, the lowest temperature can be obtained in experiments is of the order of $n K$ [10]. In this ultra-low temperature scale, the wavelength of a electron is of the order of $1 \mathrm{~mm}$, the wavelength of a hydrogen atom is of the order of $0.1 \mathrm{~mm}$; in other words, in such a case, the boundary effect can be measured in macroscale. 
In the case of ideal gases confined in annular containers or crystal lattices with periodic boundary conditions, however, we have shown that every system, very large or very small, will not show any boundary effects, that is to say even in the small systems like torus $C_{360}$ and $C_{240}$ molecules, if we can still treat such systems by the statistical method, there are no boundary effects so long as the systems have the toroidal structure. Recently, there are many studies on nanorings, especially the metal nanorings [11]. It might be expected that the method and the result of ideal gases in annular containers can be used to treat the electron gases in metal nanorings. Moreover, metal wires having diameters in the range of nanometer are very important for nanoelectronics and other nanodevice applications, and, recently, ultra-thin metal nanowires have aroused growing interest in condensed matter physics [12]. The analysis presented above applies equally to such systems.

Of special interest is the electron gas on a carbon nanotube. It is known that the electronic structure of a carbon nanotube can be either metallic or semiconducting, depending on its diameter and chirality [13]. For the metallic case we can treat the electrons on a nanotube as a free electron gas on the lateral surface of a cylinder. In such a system, along the perimeter of the transverse cross-section, even the size is very small so that the energy spectrum of the electrons is discrete, there is no boundary effect (see Sec. IV); otherwise, along the nanotube axis the boundary effect correction has to be reckoned in when the length of the nanotube is very short. With the help of the method provided in Sec. III we can calculate the thermodynamic properties of such systems directly, e.g. the specific heat in Eq. (16).

2) Long and narrow systems: Simple analysis reveals that the effect of boundary on the observable is proportional to $L / \sqrt{S}$, where $S$ is the area and $L$ the perimeter of the system. The magnitude of the factor $L / \sqrt{S}$ is determined by the geometry of the system. If the shape of the system is long and narrow, in principle, the factor $L / \sqrt{S}$ can take on an arbitrarily large value and hence the boundary effect will become significant even in macroscale.

3) Black-body radiation in small cavities: From visible light to infrared, the range of the wavelength is from 0.4 to about $10^{2}$ micron. This means that if the size of a system is of the order of micron, the boundary effect must be reckoned in. In Sec. V we have calculated the exact solution for black-body radiation in a three-dimensional rectangular cavity, from which we can estimate the influence of the boundary effect on black-body radiation.

From Eq. (40) we can estimate the influence of the boundary effect on the specific heat of a photon gas confined in a small cavity by comparing the contribution from the surface term (the second term of Eq. (40)) with the result which is obtained based on the thermodynamic limit (the first term of Eq. (40)). For this purpose, we introduce a ratio $\eta=C_{V}^{S} / C_{V}^{V}$, where $C_{V}^{S}$ is defined as the second term and $C_{V}^{V}$ the first term of Eq. (40). For the linear size of the system $L=1 \mathrm{~mm}$ and $0.1 \mathrm{~mm}$, when the temperature $T=300 \mathrm{~K}$, the ratios $\eta$ are about $1 \%$ and $10 \%$; when $T=100 \mathrm{~K}$, the ratios $\eta$ are about $3 \%$ and $30 \%$; moreover, when $T=10 \mathrm{~K}, L=1 \mathrm{~cm}$, the ratio $\eta \sim 3 \%$. This result shows that, relative to the case of particles with non-zero rest masses (whose wavelengths are relatively short), the boundary effect is significant in photon systems.

4) Systems consisting of small grains: In Sec. VI we have discussed the boundary correction to the Debye theory of the specific heat of crystals. From Eq. (55) we can learn that at low temperature the boundary effect will become significant since the contribution from the boundary term is proportional to $T^{2}$, whereas the term corresponding to the Debye's result is proportional to $T^{3}$. Therefore, the contribution from the boundary effect will become more and more important with a drop in temperature. Of course, the smaller the system size, the stronger is the influence of the boundary. Consider a macroscopic system consisting of small grains. Such a granular material can be obtained, for example, by grinding a piece of crystal to a fine powder. In such a system the volume is almost the same as the volume before grinding, whereas the total area of surface, which equals the sum of the area of all grains in the system, becomes much larger. We can rewrite the expression of the specific heat in the following form for clarity:

$$
C_{V}=\frac{V}{a^{3}} A(T)-\frac{S}{a^{2}} B(T),
$$

where the area of surface $S \sim V^{2 / 3}, A(T)$ and $B(T)$ are certain functions of the temperature. If we divide the volume $V$ up into $n$ cells of volume $V / n$, the total volume of the system is roughly speaking still $V$; however, the total area of surface becomes $n(V / n)^{2 / 3}=\sqrt[3]{n} V^{2 / 3}$. This means that the boundary effect will become important in granular materials. It can be found that before grinding the correction to the standard result (the term which is proportional to $V)$ is on the order $a / \sqrt[3]{V}$, here we have not reckoned in the influence of temperature though it is of equal importance, whereas after grinding such a correction increases to the order $\sqrt[3]{n}(a / \sqrt[3]{V})$.

5) Geometry effects vs. fluctuations: The geometry effect may be visible for small size systems. In such systems, however, the fluctuations also may not be neglected since the total number of particles $N$ is not macroscopic. A natural question that may be asked is: Can one distinguish geometry effects from the fluctuation background? Based on the results calculated above, we can estimate in what cases the geometry effects will become apparent. Next we consider some special cases as examples. 
The influence of fluctuations is on the order $\sqrt{N} / N$. In metals, take copper as an example, one $\mathrm{cm}^{3}$ contains about $10^{23}$ free electrons. The ratio $\mu_{0}^{\text {boundary }} / \mu_{0}^{\text {free }}$ reflects the influence of the boundary on the Fermi energy. When the linear size of the system is $5 \mathrm{~nm}$, the ratio $\mu_{0}^{\text {boundary }} / \mu_{0}^{\text {free }} \sim 3.3 \%$; however, in this case the fluctuation is only about $0.9 \%$; when the size of the system is $30 \mathrm{~nm}$, the ratio $\mu_{0}^{\text {boundary }} / \mu_{0}^{\text {free }} \sim 0.6 \%$, but the fluctuation is about $0.06 \%$. This means that the magnitude of the fluctuation, though also can not be neglected, is sufficiently smaller than that of the boundary effect so that one can distinguish the boundary effect in nanoscale metal systems.

For photon gases with linear size $L=1 \mathrm{~mm}$ (macroscale) and temperature $T=300 \mathrm{~K}$ (room temperature), the ratio $\eta\left(=C_{V}^{S} / C_{V}^{V}\right)$ is about $1 \%$, but, in this case, the fluctuation is negligibly small; for the case of $L=1 \mathrm{~cm}$ and $T=10 \mathrm{~K}$ the ratio $\eta \sim 3 \%$, but the fluctuation is only about $0.1 \%$. That is to say, the boundary effect can be measured in macroscopic photon systems.

6) Geometry effects vs. particle-particle interactions: In most realistic systems, there will be interactions between the particles. Next, we compare the contribution from the geometry effects with the influence of the interactions among the particles.

(a) Bose case: For dilute Bose gases, the weak interactions between particles and their low density allow for an accurate theoretical description of the effect of particle-particle interactions based on the binary collision method. When $T>T_{c}$, where $T_{c}$ is the critical temperature, the equation of state can be expressed as [14]

$$
\frac{P}{k T}=\frac{1}{\lambda^{3}} g_{5 / 2}(z)-\frac{2 a}{\lambda^{4}} g_{3 / 2}^{2}(z)+\cdots,
$$

where $a$ is the scattering length; the influence of the particle-particle interactions is described in the second term. For geometry effects, Eq. (25) gives the equation of state for an ideal Bose gas in confined space:

$$
\frac{P}{k T}=\frac{1}{\lambda^{3}} g_{5 / 2}(z)-\frac{1}{4} \frac{S}{V \lambda^{2}} g_{2}(z)+\cdots .
$$

From this result we can estimate the contributions from the geometry effects and the interparticle interactions: The leading contribution from the boundary is $-(1 / 4) S /\left(V \lambda^{2}\right) g_{2}(z)$; the leading contribution from the interparticle interaction is $-\left(2 a / \lambda^{4}\right) g_{3 / 2}^{2}(z)$. By comparing these two contributions, we can see that there exists a regime where the geometry effect dominates in an imperfect gas. Introduce the ratio between these two contributions

$$
\eta_{b}=\frac{1}{8} \frac{S \lambda^{2}}{V a} \frac{g_{2}(z)}{g_{3 / 2}^{2}(z)}
$$

where $a$ denotes the magnitude of the scattering length. The regime that the geometry effect becomes dominate can be determined by the condition $\eta_{b}>1$.

Using this result, we next compare the influence of the geometry effects with the influence of the interparticle interactions on Bose-Einstein condensation. We will show that the geometry effects, compared with the interparticle interactions, are important in some recent experiments on Bose-Einstein condensation in dilute atomic gases.

In the recent experiments on Bose-Einstein condensation, the shape of the condensate is either cigar-shaped, with a diameter about $15 \mu \mathrm{m}$ and length $300 \mu \mathrm{m} \sim 5 \mathrm{~mm}$, or approximately round with a diameter of $10 \sim 50 \mu \mathrm{m}[15,16]$. As an example, the Bose gas we considered here is near the critical point where the fugacity $z \sim 1$ and the temperature $T \sim T_{c}$, and the shape of the corresponding condensate is cigar-like (or thread-like), in other words, the shape of the system is long and narrow. In such a system, the total area of the surface is approximately equal to the area of the lateral surface, so we have

$$
d_{\max } \sim 0.12 \frac{\lambda^{2}}{a},
$$

where $d_{\max }$ is the maximum size of the transverse cross-section of the system in which the geometry effect becomes dominate. The parameters in some recent experiments have the following orders of magnitude [16]: For ${ }^{1} H$, the scattering length $a \sim 0.065 \mathrm{~nm}$, the thermal wavelength $\lambda \sim 0.25 \mu \mathrm{m}$, so we have $d_{\max } \sim 0.11 \mathrm{~mm}$; for ${ }^{7} \mathrm{Li}, a \sim-1.5 \mathrm{~nm}$, $\lambda \sim 1.2 \mu \mathrm{m}$, so $d_{\max } \sim 0.12 \mathrm{~mm}$; for ${ }^{23} \mathrm{Na}, a \sim 2.8 \mathrm{~nm}, \lambda \sim 0.26 \mu \mathrm{m}$, so $d_{\max } \sim 2.9 \times 10^{-3} \mathrm{~mm}$; for ${ }^{87} \mathrm{Rb}, a \sim 5.4 \mathrm{~nm}$, $\lambda \sim 0.45 \mu \mathrm{m}$, so $d_{\max } \sim 4.3 \times 10^{-3} \mathrm{~mm}$. From this result we can see that, compared with the interparticle interactions, the geometry effects can not be ignored in the experiments on Bose-Einstein condensation of ${ }^{1} \mathrm{H}$ and ${ }^{7} \mathrm{Li}$. For example, in the experiment on Bose-Einstein condensation of atomic hydrogen the condensate is $15 \mu \mathrm{m}$ in diameter and $5 \mathrm{~mm}$ in length [16], so the ratio $\eta_{b} \sim 7.5$, i.e., the geometry effects are more important than the interparticle interactions in the experiment on Bose-Einstein condensation of atomic hydrogen.

Strictly speaking, in the recent experiments on Bose-Einstein condensation the atomic gases are trapped in magnetic traps rather than boxes with hard walls. Usually, such traps are approximately described as effective three-dimensional 
harmonic wells cylindrically symmetric about the $z$-axis. In above analysis we assume that the Bose gases are trapped in hard-wall tubes, i.e., for simplifying the treatment, we replace the harmonic wells by tubes, but the result, to orders of magnitude, is reliable.

(b) Fermi case: For an imperfect Fermi gas the equation of state is [14]

$$
\frac{P}{k T}=(2 j+1) \frac{1}{\lambda^{3}} f_{5 / 2}(z)-2 j(2 j+1) \frac{a}{\lambda^{4}} f_{3 / 2}^{2}(z)+\cdots,
$$

where $j$ is the spin of the particle. Like the treatment in the Bose case, we can also compare the geometry effects with the interparticle interactions by comparing the second terms in Eqs. (25) and (61) directly. Next, as an example, we compare these two influences on the ground state of a Fermi system.

The ground-state energy for an imperfect Fermi gas is given by $(j=1 / 2)[14,17]$

$$
E=\frac{3}{5} N \frac{\hbar^{2}}{2 m}\left(3 \pi^{2} \frac{N}{V}\right)^{2 / 3}+N \frac{\hbar^{2}}{2 m} 2 \pi a \frac{N}{V}+\cdots
$$

the contribution from the interparticle interactions described in the second term. The corresponding chemical potential is

$$
\begin{aligned}
\mu_{0} & =\frac{\hbar^{2}}{2 m}\left(3 \pi^{2} \frac{N}{V}\right)^{2 / 3}+\frac{\hbar^{2}}{2 m}\left(4 \pi a \frac{N}{V}\right) \\
& =\mu_{0}^{\text {free }}+\mu_{0}^{\text {interparticle }}
\end{aligned}
$$

where $\mu_{0}$ is equal to the Fermi energy of the ideal Fermi gas plus the second term which describes the contribution from the interparticle interactions. Similarly, as expressed in Eq. (28), the Fermi energy containing the contribution from the boundary can be written as $\mu_{0}=\mu_{0}^{\text {free }}+\mu_{0}^{\text {boundary }}$. Introducing a ratio $\eta_{f}=\mu_{0}^{\text {boundary }} / \mu_{0}^{\text {interparticle }}$, we can compare the corrections due to the geometry effects with the corrections that emerge from the interactions among the particles. Direct calculation gives

$$
\eta_{f}=\frac{1}{16}\left(3 \pi^{2}\right)^{1 / 3} \frac{1}{a} \frac{S}{V}\left(\frac{N}{V}\right)^{-2 / 3}
$$

Taking a box of side-length $d$ as an example, we have $\eta_{f}=(3 / 8)\left(3 \pi^{2}\right)^{1 / 3} l^{2} /(a d)$, where $l=(V / N)^{1 / 3}$ is the mean interparticle distance. Obviously, if $\eta_{f}>1$, compared with the interparticle interactions, the geometry effects will dominate in the system. $\eta_{f}>1$ gives the condition

$$
\frac{d}{l}<1.160 \frac{l}{a}
$$

This condition is equivalent to the requirement of low densities and small system sizes. That is to say, in a small dilute Fermi gas, the influence of geometry effects will exceed the influence of interparticle interactions.

Furthermore, in this paper we also consider the geometry effects on photon gases and on phonon gases. The photon gas can be regarded as a genuine ideal gas in statistical mechanics since the cross section of photon-photon scattering, which is only a loop correction, is exceedingly small. Therefore, there is no contribution from the interparticle interaction. In the harmonic approximation, the phonon gas can also be regarded as an ideal gas. That is to say, in photon gases and in phonon gases the contributions from the geometry effects are the main corrections to the standard results.

\section{DISCUSSIONS AND CONCLUSIONS}

The key problem in statistical mechanics is to calculate the partition function, i.e., to solve the sum over all possible states. Often, this sum is difficult to calculate. To calculate the partition function approximately, in the thermodynamic limit $V \rightarrow \infty$, one can convert the sum to an integral by introducing a state density. In so doing, however, the information about the system geometry has been lost because the approximation that the spectrum is continuous has been taken during this process. The information about the system geometry is embodied in the structure of the spectrum, so if we want to involve the geometry information in the thermodynamic quantities, we have to solve the sum over the states directly. We have developed two methods to solve the sum: One is an approximate 
method which can be used to deal with the system with an irregular boundary [4]. Another method considered in the present paper is based on the Euler-MacLaurin formula; sometimes this method leads to exact solutions. However, the prerequisite for applying the Euler-MacLaurin formula is that the spectrum is already known.

The method for performing the summation by the Euler-MacLaurin formula is easy to apply to the cases of ideal gases in external potentials and nonideal gases if the spectrum of the system is already known. Fortunately, the spectrum can be obtained by using many systematic methods exactly or approximately. Such cases we will discuss in detail elsewhere [18].

Intrinsically the boundary effect arises from the interaction between the particles and the boundary. The influence of this interaction can be embodied in boundary conditions. The fix-end boundary condition, like the case of a particle in an infinite depth potential, reflects the interaction between the particles and the wall of the box. However, if there is no boundary, of course, there is no interaction between the particles and the boundary; in other words, there is no boundary effect. In such a case we may apply periodic boundary conditions, and then the boundary terms will vanish in the exact solution.

From the results given above, we can see that the grand potential of a system in confined space is less than that in free space since the sign of the leading geometry term, e.g., the second term in Eq. (20), is negative. It means that the existence of a boundary tends to reduce the number of states of the system, in other words, to reduce the volume of the phase space - the set of all possible states. This is just because, for ideal gases, in free space the spectrum is continuous while in confined space the spectrum gets discrete. Thus the number of modes in confined space is less than that in free space.

The boundary term which is proportional to the area of the surface is always negative (the other terms corresponding to geometry effects are usually negligible). A natural question that may be asked is: What will happen when the contribution from the boundary effect become large enough, e.g., in the case of the size of the system is very small or the temperature is very low, so that the grand potential is less than zero? The answer is that in such a case the statistical method is no longer valid because the grand potential is the logarithm of the grand partition function and a negative grand potential means that the number of the states is less than one.

In conclusion, by using the Euler-MacLaurin formula, the geometry effects on the statistical mechanics of various systems, such as ideal Bose and Fermi gases, photon gases, phonon gases, are discussed. From the exact solutions we can see that the grand potential can be expressed as a sum of two parts: one is just the result obtained in the thermodynamic limit $V \rightarrow \infty$; another is the contribution from the geometry effect. The latter is consisted of a series of terms: the first term, by comparing with the result given in Ref. [4], reflects the influence of the shape of the boundary; the second term, which is proportional to the Euler-Poincaré characteristic number, reflects the influence of the topological property of the system; the rest terms are always negligible and the number of such terms is finite when the problem is exactly solvable, or else it may be infinite. Moreover, we discuss the validity of the approximate method for calculating the effects of boundary and connectivity, presented in Ref. [4], which is based on the mathematical work given by Kac [6] and can be used to deal with the ideal gas system with an irregular boundary in two dimensions. We also analyze the possible geometry effects in realistic systems. It is hoped that the geometry effects can be encountered in the regime of mesoscopic scale. For photon gases, the geometry effects may be observed even in macroscale systems. In imperfect gases, we compare the geometry effects with the interparticle interactions and point out that there exists a regime where the geometry effects dominate in interacting systems.

We are very indebted to Dr. G. Zeitrauman for his encouragement. This work is supported in part by LiuHui fund.

\section{APPENDIX A: THERMODYNAMIC QUANTITIES IN THREE-DIMENSIONAL BOXES}

Following general procedures, we can obtain the thermodynamic quantities for an ideal gas in a three-dimensional box although the geometry terms in Eq. (25) make the derivation get tedious.

The equation of state:

$$
\begin{aligned}
\frac{P V}{k T} & =\ln \Xi=\frac{V}{\lambda^{3}} h_{5 / 2}(z)-\frac{1}{4} \frac{S}{\lambda^{2}} h_{2}(z)+\frac{1}{16} \frac{L}{\lambda} h_{3 / 2}(z)-\frac{1}{8} h_{1}(z), \\
N & =z \frac{\partial}{\partial z} \ln \Xi=\frac{V}{\lambda^{3}} h_{3 / 2}(z)-\frac{1}{4} \frac{S}{\lambda^{2}} h_{1}(z)+\frac{1}{16} \frac{L}{\lambda} h_{1 / 2}(z)-\frac{1}{8} h_{0}(z) .
\end{aligned}
$$

Internal energy:

$$
\frac{U}{N k T}=\frac{3}{2} \frac{h_{5 / 2}(z)}{h_{3 / 2}(z)}(1+\Delta)+\frac{1}{N}\left[-\frac{1}{4} \frac{S}{\lambda^{2}} h_{2}(z)+\frac{1}{32} \frac{L}{\lambda} h_{3 / 2}(z)\right]
$$


where

$$
\Delta=\frac{1}{N}\left[\frac{1}{4} \frac{S}{\lambda^{2}} h_{1}(z)-\frac{1}{16} \frac{L}{\lambda} h_{1 / 2}(z)+\frac{1}{8} h_{0}(z)\right]
$$

Specific heat:

$$
\begin{aligned}
\frac{C_{V}}{N k}= & {\left[\frac{15}{4} \frac{h_{5 / 2}(z)}{h_{3 / 2}(z)}-\frac{9}{4} \frac{h_{3 / 2}(z)-\delta_{1}}{h_{1 / 2}(z)-\delta_{2}}\right](1+\Delta) } \\
& -\frac{1}{2} \frac{1}{N} \frac{S}{\lambda^{2}}\left[h_{2}(z)-\frac{3}{4} h_{1}(z) \frac{h_{3 / 2}(z)-\delta_{1}}{h_{1 / 2}(z)-\delta_{2}}\right]+\frac{3}{64} \frac{1}{N} \frac{L}{\lambda}\left[h_{3 / 2}(z)-h_{1 / 2}(z) \frac{h_{3 / 2}(z)-\delta_{1}}{h_{1 / 2}(z)-\delta_{2}}\right],
\end{aligned}
$$

where

$$
\begin{gathered}
\delta_{1}=\frac{1}{6} \frac{S \lambda}{V} h_{1}(z)-\frac{1}{48} \frac{L \lambda^{2}}{V} h_{1 / 2}(z), \\
\delta_{2}=\frac{1}{4} \frac{S \lambda}{V} h_{0}(z)-\frac{1}{16} \frac{L \lambda^{2}}{V} h_{-1 / 2}(z)+\frac{1}{8} \frac{\lambda^{3}}{V} h_{-1}(z) .
\end{gathered}
$$

We also have

$$
\frac{\partial z}{\partial T}=-\frac{3}{2} \frac{z}{T} \frac{h_{3 / 2}(z)-\delta_{1}}{h_{1 / 2}(z)-\delta_{2}}
$$

[1] H. Potempa and L. Schweitzer, J. Phys. C10, L431 (1998).

[2] D. Braun, G. Montambaux and M. Pascaud, Phys. Rev. Lett. 81, 1062 (1998).

[3] V. E. Kravtsov and V. I. Yudson, Phys. Rev. Lett. 82, 157 (1999).

[4] W.-S. Dai and M. Xie, Phys. Lett. A311, 340 (2003).

[5] M. Abramowitz, and C. A. Stegun (Eds.). Handbook of Mathematical Functions with Formulas, Graphs, and Mathematical Tables, (Dover, New York, 1972).

[6] M. Kac, Am. Math. Monthly 73, 1 (1966).

[7] K. Huang, Statistical Mechanics, (2nd ed., John Wiley \& Sons, New York, 1987).

[8] C. Kittel, Introduction to Solid State Physics, (7nd ed., John Wiley \& Sons, New York, 1996).

[9] Y. Imry, Introduction to Mesoscopic Physics, (Oxford University Press, 1997).

[10] A. E. Leanhardt, T. A. Pasquini, M. Saba, A. Schirotzek, Y. Shin, D. Kielpinski, D. E. Pritchard, and W. Ketterle, Science 301, 1513 (2003).

[11] V. M. K. Bagci, O. Gülseren, T. Yildirim, Z. Gedik, and S. Ciraci, Phys. Rev. B66, 045409 (2002).

[12] S. Ciraci, A. Buldum and Inder P. Batra, J. Phys.: Condens. Matter 13, R537 (2001).

[13] R. Saito, M. Fujita, G. Dresselhaus, M. S. Dresselhaus, Appl. Phys. Lett. 60, 2204 (1992); J. W. Mintmire, B. I. Dunlap, C. T. White, Phys. Rev. Lett. 68, 631 (1992); N. Hamada, S. Sawada, A. Oshiyama, Phys. Rev. Lett. 68, 1579 (1992); J. W. G. Wilder, L. C. Venema, A. G. Rinzler, R. E. Smalley, C. Dekker, Nature 391, 59 (1998); T. W. Odom, J.-L. Huang, P. Kim, C. M. Lieber, Nature 391, 62 (1998).

[14] T. D. Lee and C. N. Yang, Phys. Rev. 105, 1119 (1957).

[15] J. R. Anglin, W. Ketterle, Nature 416, 211 (2002).

[16] D. Kleppner, T. J. Greytak, T. C. Killian, D. G. Fried, L. Willmann, D. Landhuis, and S. C. Moss, in Proceedings of the International School of Physics "Enrico Fermi" Course CXL, ed. M. Inguscio, S. Stringari and C. Wieman (IOS Press, Amsterdam 1999); D. G. Fried, T. C. Killian, L. Willmann, D. Landhuis, S. C. Moss, D. Kleppner, and T. J. Greytak, Phys. Rev. Lett., 81, 3811 (1998).

[17] K. Huang, C. N. Yang, Phys. Rev. 105, 767 (1957); T. D. Lee, K. Huang, C. N. Yang, Phys. Rev. 106, 1135 (1957).

[18] W.-S. Dai and M. Xie, in preparation. 